\title{
ADAPTACIÓN CONTINUA Y MEJORAS EN LA EDICIÓN DE RELIEVE
}

\section{( Continuous adaptation and improvement in the edition of RELIEVE) por}

$\underline{\text { Article record }}$
$\underline{\text { About authors }}$
$\underline{\text { HTML format }}$

\author{
Francisco M. Aliaga \\ (Francisco.Aliaga@uv.es) \\ $\mathrm{y}$ \\ Jesús Suárez Rodríguez \\ (rodrigus@uv.es )
}

$\underline{\text { Ficha del artículo }}$

$\underline{\text { Sobre los autores }}$

Formato HTML

Abstract
The changes in RELIEVE (Electronic Journal of Educa-
tional Research, Assessment and Evaluation) during the
last year are presented and commented.
Keywords
Scholarly Journals; Electronic Journals; Electronic Pub-
lishing; Information Dissemination; Internet; Primary
Sources; Users (Information)

Resumen
$\begin{aligned} & \text { Se revisan lo cambios en la evolución de la Revista } \\ & \text { Electrónica de Investigación y Evaluación Educativa } \\ & \text { (RELIEVE) durante el último año. }\end{aligned}$
Descriptores
Revistas académicas; Revistas Electrónicas; Edición Elec-
trónica; Comunicación de la Información; Internet;
Fuentes Primarias

\section{Presentación}

Hace ahora un año tomamos ilusionadamente las riendas de la Revista ELectrónica de Investigación y EValuación Educativa (RELIEVE), inaugurando así una nueva época de la misma (Aliaga y Suárez, 2002). Como ya comentamos entonces, teníamos un edificio construido sobre sólidos cimientos, por lo que nuestro empeño era asentar y difundir este proyecto pionero, a nuestro entender tan interesante.

Ha sido una año repleto de actividad y de novedades, destinadas todas ellas a incrementar, aún más, la calidad de esta revista académica que, repetimos con orgullo indisimulado, es la primera revista electrónica de España y que tiene una indudable vocación internacional. Queremos en este texto comentar alguna de las novedades planteadas en RELIEVE durante el año transcurrido.

La primera ha sido el cambio de ubicación del servidor y, por tanto, de la dirección electrónica de la revista, que ha pasado de estar en la Universidad de Cádiz, donde nació esta publicación, a su nueva dirección en la Universidad de Valencia (www.uv.es/RELIEVE), debido a motivos técnicos. Para que no se perdiese ningún enlace ya existente a la revista se insta- 
laron en la antigua dirección unos programas que redireccionan automáticamente al visitante a la nueva ubicación. Hubo que hacer también un trasvase de toda la información disponible, de modo que se hicieron nuevas versiones electrónicas de todos y cada uno de los artículos publicados en la revista desde su origen, manteniendo el número de visitas en los contadores y especificando, en un nuevo encabezamiento, la antigua dirección y la nueva.

A fin de aprovechar mejor los avances que han ido apareciendo en el diseño de páginas web se construyó un nuevo entorno gráfico, basado en frames, que ha supuesto un cambio en la apariencia de la revista. Se ha incluido en la parte superior una zona fija de identificación, en la que aparecen el nuevo logotipo (basado en el de la asociación matriz, AIDIPE) y el nombre de la revista (en español y en inglés), de modo que siempre esté presente para los lectores. En la zona izquierda se ha incluido un menú de navegación por las distintas partes de la revista. Cuando se pica en ellas aparece el objetivo en la zona principal, que por otro lado puede visualizarse de manera independiente como página a pantalla completa.

Se está procediendo a desarrollar para cada pantalla de información de la revista una presentación bilingüe en español e inglés (la actual lingua franca de la ciencia) que, habida cuenta de las facilidades de comunicación que aporta internet, permita una difusión no sólo a la comunidad hispana, sino a una población de potenciales lectores interesados aún más amplia.

En los aspectos formales también se ha cambiado, en consonancia con lo anterior, la apariencia de los artículos. Se ha sistematizado la presentación de los artículos (titulo, resumen y palabras claves) tanto en español como en inglés para cada artículo. En el encabezamiento de cada uno aparece la referencia completa del artículo, de modo que se facilite (mediante el "recorto y pego") la cita de los trabajos publicados en RELIEVE, así como su fácil identificación. Hemos continuado con la práctica, ya iniciada en la etapa anterior de RELIEVE por su antiguo director ejecutivo, Gregorio Rodríguez, de instalar un contador específico que mida la difusión diferencial de todos y cada uno de los artículos publicados. A ello hemos añadido, al final de cada uno de los artículos, una conexión a nedstat (www.nedstat.com), una de las principales y más prestigiosas instituciones de estadísticas de visitas a páginas web de internet, teniendo así una auditoria externa sobre países de origen de las visitas, frecuencia, distribución temporal, etc.). Creemos que esta medida aporta un control de la difusión de extraordinaria importancia para avalar no ya sólo la calidad de la revista, sino también la de cada uno de los artículos por separado, permitiendo la construcción de perfiles de los usuarios interesados en cada uno de los textos, yendo así más allá de las clásicas medidas de impacto por revista que utilizan, por ejemplo, el Journal Citation Report del Institute for Scientific Information, y que de manera tan inadecuada se utilizan a veces para evaluar el trabajo específico de los autores (Garfield, 1998; López Piñero y Terrada,1992; Fernández Cano, 1995; Aliaga y Orellana, 1999, 2001).

En cada uno de los artículos se ha incluido un conjunto de meta datos (oculto para el simple lector) con la intención de facilitar la localización de los textos. Se ha optado por el código Dublin Core, uno de los que tienen una mayor implantación en el ámbito de las publicaciones académicas.

En la parte superior de cada uno de los artículos se han incluido unas pequeñas "ventanitas", con fondo azul claro, que aportan un valor añadido a los textos, permitiendo aprovechar las posibilidades que ofrece el hipertexto. Así, aparecen enlaces a otros artículos relacionados, la posibilidad de enviar, con un sólo clic, comentarios a cada uno de los artículos, la posibilidad de imprimir directamente el artículo (y sólo el artículo, sin el problema de manejar los frames), enlace a la ficha de presentación de los autores, etc. Estas ventanas aparecen tanto en español (lado derecho) como en inglés (en el lado izquierdo). 
Aliaga, F.M. y Suárez Rodríguez, J. (2003). Adaptación continua y mejoras en la edición de RELIEVE. RELIEVE:, v. 9, n. 1, p. 3-9. http://www.uv.es/RELIEVE/v9n1/RELIEVEv9n1_0.htm

Desde tales ventanas hipertextuales puede accederse también a la versión de los artículos en formato PDF. Se trata éste de un formato de gran implantación en internet que aporta varias ventajas. Por un lado, unifica la presentación, ya que ese formato se ve igual en cualquier ordenador y con cualquier sistema operativo. Igualmente aporta la paginación que, en su caso, podría facilitar las citas de textos concretos de un artículos.

Finalmente, dentro de los aspectos formales de los artículos, hemos añadido al final de cada artículo, una ficha con los datos fundamentales del artículos, con la intención de facilitar su clasificación y eventual inclusión en diferentes Bases de Datos.

\section{Datos del último volumen}

A lo largo de 2002 se ha publicado el volumen 8 de RELIEVE. Hemos añadido un índice conjunto de todo el volumen (sus dos números semestrales) (http://www.uv.es/RELIEVE/indexv8.htm) .

El Comité Editorial decidió mantener la cantidad de artículos publicados estable para los dos números (cuatro artículos), aunque en el primero se incluyó además un artículo editorial de presentación de la Segunda Época de la revista. Sin embargo ha de hacerse notar que el número de manuscritos recibidos se incrementó apreciablemente para el segundo semestre respecto al primero (de 7 pasó a 10), tal y como se aprecia en la Tabla 1 que podemos ver a continuación.

Gráfico 1 - Variación en los artículos recibidos y publicados

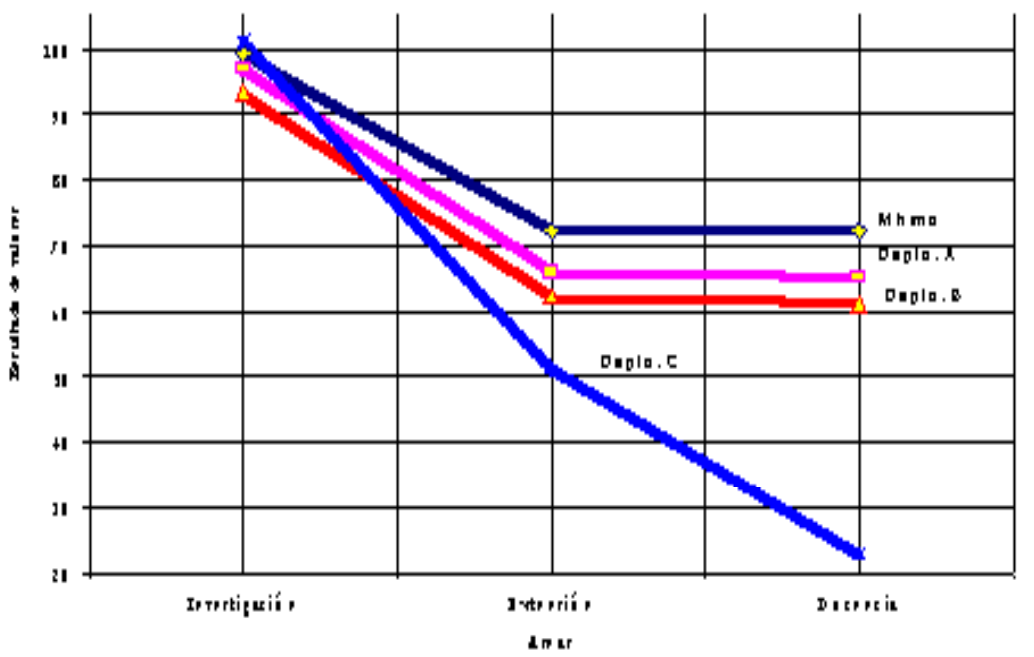

A partir de los datos comentados podemos calcular fácilmente el porcentaje de artículos rechazados (o retirados por los autores tras recibir el informe de los revisores) que fue del $42,9 \%$ en el número 1 y pasó al $60 \%$ en el segundo (dando un $53 \%$ para el total del volumen 8 ). Creemos que estos datos son una muestra evidente del alto nivel de exigencia al que el proceso de revisión ciega por pares adoptado por RELIEVE somete a los manuscritos recibidos, garantía a su vez de un elevado nivel de calidad y del interés por publicar en nuestra revista por parte de los autores.

En buena medida la calidad de una revista se debe no sólo a la política editorial adoptada y, desde luego, al trabajo de los autores que envían sus trabajos, sino a la calidad de la tarea de los revisores. RELIEVE se siente orgullosa y agradecida al amplio elenco de especialistas que han contribuido desinteresadamente con su tiempo, sus conocimientos y su amabilidad a 
Aliaga, F.M. y Suárez Rodríguez, J. (2003). Adaptación continua y mejoras en la edición de RELIEVE. RELIEVE:, v. 9, n. 1, p. 3-9. http://www.uv.es/RELIEVE/v9n1/RELIEVEv9n1_0.htm

una labor frecuentemente oscura, pero imprescindible. En la edición del primer número del volumen 8 participaron doce revisores, mientras que fueron catorce en el segundo. Hay que tener en cuenta que algunos artículos fueron directamente rechazados por el Comité Editorial, al juzgar que no se adecuaban a la Política Editorial de RELIEVE, por lo que no llegó a participar ningún revisor. En total, para los artículos finalmente publicados participaron diez revisores (en el número 1) y nueve (en el número 2), lo que hace una media de 2,5 revisores por artículo en el primer semestre (en algún artículo llegamos a recurrir a cuatro distintos) y 2,25 en el segundo, lo que da una media de 2,38 revisores por artículo publicado para en conjunto del volumen 8 .

El objetivo que nos hemos planteado es que el proceso de revisión y edición de un artículo desde que nos llega el manuscrito original hasta que sale publicado en la revista sea de apenas un mes. Se trata, sin duda, de un objetivo muy ambicioso, sobre todo si lo comparamos con los plazos de publicación en revistas impresas. Pero creemos que la agilidad en la respuesta es una de las principales bazas de las revistas electrónicas (Rodríguez, 1999; Aliaga y Suárez, 2002). Por ello hemos instaurado el sistema de edición continua, de modo que cada artículo sale publicado en cuanto el Comité Editorial, a la vista de los informes de los revisores, lo aprueba, sin tener que esperar a tener otros artículos de ese mismo número en idéntica situación. Este concepto flexibiliza la noción de "número" de una revista, que se mantiene fundamentalmente por razones formales.
En el número correspondiente al primer semestre de 2002, el proceso de edición más largo (periodo que transcurre entre la recepción y la publicación de un artículo y que incluye la revisión por jueces externos, rectificación por parte del autor, nueva revisión en su caso, maquetación, comprobación de galeradas por los autores e inclusión en los distintos índices de la revista) fue de 5,3 meses, mientras que el más corto fue de apenas 0,2 días (referido en éste caso a un artículo rechazado directamente por el Comité Editorial), dando una media para el conjunto de todos los manuscritos de 2,1 meses, que pasa a ser de 3 meses si nos referimos exclusivamente a los artículos que fueron finalmente aprobados y publicados (es decir, sin incluir los rechazos rápidos). Creemos que aunque no alcanza todavía nuestro objetivo sí son unas cifras notablemente inferiores al de otras revistas impresas. Con el rodaje y el mejor ajuste del Comité Editorial estas cifras mejoraron para el segundo número del volumen 8 . Así, el proceso de revisión más largo fue de 4 meses, y el más corto de 0,1 , siendo la media de duración del proceso de edición para todos los manuscritos de ese segundo semestre de 1,9 meses, y de sólo 2 meses para los artículos que finalmente se han publicado. Confiamos en seguir mejorando estos plazos, aunque no siempre está en nuestra mano, pues depende de unos revisores muy ocupados, la presteza de los autores en realizar las modificaciones o justificar sus argumentos, etc.

Tabla 1 - Duración proceso de edición en RELIEVE

\begin{tabular}{|l|c|c|c|}
\cline { 2 - 4 } \multicolumn{1}{c|}{} & Vol.8 n1 & Vol.8 n2 & Total Vol. 8 \\
\hline Duración del proceso de edición más largo & 5,3 & 4 & 5,3 \\
\hline Duración del proceso de edición más corto & 0,2 & 0,1 & 0,1 \\
\hline Media de duración de la edición total manuscritos & 2,1 & 1,9 & 2 \\
\hline Media de duración de la edición artículos publicados & 3 & 2 & 2,76 \\
\hline
\end{tabular}

Duración medida en meses 
Aliaga, F.M. y Suárez Rodríguez, J. (2003). Adaptación continua y mejoras en la edición de RELIEVE.

RELIEVE:, v. 9, n. 1, p. 3-9. http://www.uv.es/RELIEVE/v9n1/RELIEVEv9n1_0.htm

También el nivel de internacionalización de la revista ha ido progresando paulatinamente. Así, para el número primero un total del $28,6 \%$ de los manuscritos recibidos procedían de fuera de España, valor éste que ascendió al 50\% para el número correspondiente al segundo semestre, prueba del creciente interés por nuestra publicación, que trasciende las fronteras. Creemos que la facilidad de comunicación del medio electrónico en el que se publica RELIEVE, así como su creciente difusión e impacto, van dando un perfil cada vez más internacional a esta publicación, tendencia ésta que habría que confirmar en volúmenes posteriores.

\section{Datos sobre difusión, impacto y calidad}

Son variados los datos, procedentes de distintas fuentes, que indican que la difusión de RELIEVE está teniendo igualmente un notable incremento. Al inicio del año 2002 el contador de visitas de la página principal sobrepasaba ligeramente las 60.000 visitas, número notable para una publicación académica especializada. Hay que señalar, además, que ese número sólo cubre las visitas a la página principal de la revista (cada artículo tiene, como decíamos antes, su propio contador específico) y que no incluye todas las recibidas por ella, ya que hasta Febrero de 1997 no se puso en marcha esta utilidad. En cualquier caso, durante el año 2002 la tasa de visitas se ha acelerado, sobrepasando las 100.000 antes de fin de año.

Este análisis, exclusivamente contable, no nos permitía realizar un estudio sobre las zonas de procedencia, por lo que a lo largo del primer semestre de 2003 añadimos a la página principal dos enlaces con distintos servicios de estadísticas de visitas. A pesar de la distinta metodología empleada por cada uno de estos servicios (nedstat e hispastats), podemos afirmar que apenas un tercio de las visitas proceden de España, un porcentaje ligeramente inferior proceden de México y el resto de otros países, fundamentalmente hispano y norteamericano.

Un índice específico que permite medir la difusión de las revistas electrónicas es el número de instituciones de prestigio que enlazan con la revista. En el caso de RELIEVE dichas instituciones son muy numerosas e incluyen organizaciones tan prestigiosas como la AERA, ERIC, EERA, etc. así como una gran cantidad de bibliotecas universitarias.

Hay otros índices y Bases de Datos que vienen incluyendo a RELIEVE, lo que indica una difusión y un reconocimiento internacional importante. No obstante aún es mucho lo que queda por hacer en esta tarea, de lo que daremos cuenta en números posteriores.

En resumen, la Revista ELectrónica de Investigación y EValuación Educativa (RELIEVE) sigue en un proceso de actualización y mejora continua, en su deseo de convertirse en una publicación de interés, utilidad y prestigio en el ámbito educativo. Todo ello lo conseguiremos gracias a la colaboración conjunta de los autores, los revisores y, no lo olvidemos, los lectores. A todos ellos nuestra gratitud más sincera.

\section{Referencias}

Aliaga, F. y Orellana, F.(2001).Análisis de estabilidad del Journal Citation Report y su implicación como requisito para la evaluación de la calidad de las publicaciones sobre investigación educativa: problemas y limitaciones. En AIDIPE, Investigación y evaluación educativas en la Sociedad del Conocimiento, 339-344. La Coruña: AIDIPE.

Aliaga, F. y Orellana, N. (1999). La utilización de las bases de datos del ISI para la evaluación de la calidad de las publicaciones sobre investigación educativa en España: argumentos para un debate. En AIDIPE, Nuevas realidades educativas, nuevas necesidades metodológicas, pp. 29-34. Málaga: CEDMA

Aliaga, F. y Suárez Rodríguez, J. (2002). Tendencias actuales en la edición de revistas electrónicas: nueva etapa en RELIEVE. Revista ELectrónica de Investigación y EValuación Educativa, v. 8, n. 1. Consultado el 8 de Febrero de 2003 en http://www.uv.es/RELIEVE/v8n1/RELIEVEv $\underline{8 \mathrm{n} 10 . \mathrm{htm}}$. 
Aliaga, F.M. y Suárez Rodríguez, J. (2003). Adaptación continua y mejoras en la edición de RELIEVE. RELIEVE:, v. 9, n. 1, p. 3-9. http://www.uv.es/RELIEVE/v9n1/RELIEVEv9n1 0.htm

Fernández Cano, A.(1995). Métodos para evaluar la investigación en psicopedagogía. Madrid: Síntesis.

Garfield,E. (1998). The use of Journal Impact Factors and citation analysis for evaluation of science. Presented At the 41st Annual Meeting of the Council of Biology Editors, Salt Lake City, UT, May 4, 1998 - April 17. Consultado el 10 de Febrero de 2003 en http://www.garfield.library.upenn.edu/papers/ev al of science CBE(Utah).html

López Piñero, J.M. y Terrada, M.L. (1992). Los indicadores bibliométricos y la evaluación de la actividad médico científica.(II). Los indicadores de producción, circulación y dispersión, consumo de la información y repercusión. Medicina Clínica, vol 98, 42-148.

Price, D.J.S. (1963). Little Science, Big Science. New York: Columbia University Press.

Rodríguez, G. (1999). Revistas electrónicas: Cybernautas y/o papirófilos. Cambios en la comunicación científica. Revistas de Investigación Educativa, Vol 17, n² 2, pp. 491-494.

\section{ABOUT THE AUTHORS / SOBRE LOS AUTORES}

Francisco M. Aliaga (Francisco.Aliaga@uv.es): Es Profesor Titular en el Departamento de Métodos de Investigación y Diagnóstico en Educación de la Universidad de Valencia (España), cuya dirección es Avda. Blasco Ibáñez, 30. 46010-Valencia. Sus principales líneas de trabajo son "Internet y Educación" y "Metodología de investigación". La dirección de su página Web es www.uv.es/ aliaga. Fue nombrado Director Ejecutivo de RELIEVE en Septiembre de 2001.

Jesús M. Suárez Rodriguez (rodrigus@uv.es): Es Profesor Titular en el Departamento de Métodos de Investigación y Diagnóstico en Educación de la Universidad de Valencia (España), cuya dirección es Avda. Blasco Ibáñez, 30. 46010-Valencia. Sus principales líneas de trabajo son "Internet y Educación" y "Metodología de investigación". Fue nombrado Director de RELIEVE en Septiembre de 2001. 
Aliaga, F.M. y Suárez Rodríguez, J. (2003). Adaptación continua y mejoras en la edición de RELIEVE.

RELIEVE:, v. 9, n. 1, p. 3-9. http://www.uv.es/RELIEVE/v9n1/RELIEVEv9n1_0.htm

\section{ARTICLE RECORD / FICHA DEL ARTÍCULO}

\begin{tabular}{|c|c|}
\hline $\begin{array}{l}\text { Reference / } \\
\text { Referencia }\end{array}$ & $\begin{array}{l}\text { Aliaga, F. y Suárez Rodríguez, J (2003). Adaptación continua y mejoras en la edición } \\
\text { de RELIEVE. Revista ELectrónica de Investigación y EValuación Educativa, v. 9, n. } \\
\text { 1. http://www.uv.es/RELIEVE/v9n1/RELIEVEv9n1_0.htm. Consultado en (poner } \\
\text { fecha). }\end{array}$ \\
\hline Title / Título & $\begin{array}{l}\text { Adaptación continua y mejoras en la edición de RELIEVE [Continuous adaptation } \\
\text { and improvement in the edition of RELIEVE] }\end{array}$ \\
\hline Authors / Autores & Francisco M. Aliaga y Jesús M. Suárez Rodríguez \\
\hline $\begin{array}{l}\text { Review / } \\
\text { Revista }\end{array}$ & Revista ELectrónica de Investigación y EValuación Educativa (RELIEVE), v. 9, n. 1 \\
\hline ISSN & $1134-4032$ \\
\hline $\begin{array}{l}\text { Publication date / } \\
\text { Fecha de publicación }\end{array}$ & 2003 \\
\hline $\begin{array}{l}\text { Abstract / } \\
\text { Resumen }\end{array}$ & $\begin{array}{l}\text { The changes in RELIEVE (Electronic Journal of Educational Research, Assessment } \\
\text { and Evaluation) during the last year are presented and commented } \\
\text { Se revisan lo cambios en la evolución de la Revista Electrónica de Investigación y } \\
\text { Evaluación Educativa (RELIEVE) durante el último año. }\end{array}$ \\
\hline $\begin{array}{l}\text { Keywords } \\
\text { Descriptores }\end{array}$ & $\begin{array}{l}\text { Scholarly Journals; Electronic Journals; Electronic Publishing; Information Dissemi- } \\
\text { nation; Internet; Primary Sources; Users (Information) } \\
\text { Revistas académicas; Revistas Electrónicas; Edición Electrónica; Comunicación de la } \\
\text { Información; Internet; Fuentes Primarias }\end{array}$ \\
\hline $\begin{array}{l}\text { Institution / } \\
\text { Institución }\end{array}$ & Valencia University (Spain) \\
\hline $\begin{array}{l}\text { Publication site / } \\
\text { Dirección }\end{array}$ & http://www.uv.es/RELIEVE \\
\hline Language / Idioma & Español (Title, abstract and keywords in english) \\
\hline
\end{tabular}

\section{Revista ELectrónica de Investigación y EValuación Educativa (RELIEVE)}

[ ISSN: 1134-4032 ]

(C) Copyright 2002, RELIEVE. Reproduction and distribution of this articles it is authorized if the content is no modified and their origin is indicated (RELIEVE Journal, volume, number and electronic address of the document).

// C Copyright 2002, RELIEVE. Se autoriza la reproducción y distribución de este artículo siempre que no se modifique el contenido y se indique su origen (RELIEVE, volumen, número y dirección electrónica del documento). 